Editorial Article

Open Access

\title{
Manual Lymphatic Drainage in Autism Treatment
}

\author{
Nicola Antonucci ${ }^{1}$, Stefania Pacini ${ }^{2}$ and Marco Ruggiero ${ }^{2 \star}$ \\ ${ }^{1}$ Biomedical Centre for Autism Research and Treatment, Bari, Italy \\ ${ }^{2}$ Silver Spring Sagl, Arzo-Mendrisio, Switzerland
}

\section{Article Info}

\author{
*Corresponding author: \\ Marco Ruggiero \\ Silver Spring Sagl \\ Via Raimondo Rossi 24, Arzo-Mendrisio \\ 6864 \\ Switzerland \\ Tel: +41792309283 \\ E-mail:marco.drruggiero@gmail.com
}

Received: December 11, 2018

Accepted: December 22, 2018

Published: December 28, 2018

Citation: Antonucci N, Pacini S, Ruggiero M. Manual Lymphatic Drainage in Autism Treatment. Madridge J Immunol. 2018; 3(1): 69-72.

doi: $10.18689 / \mathrm{mjim}-1000116$

\section{Copyright: @ $\odot 2018$ The Author(s). This work is licensed under a Creative Commons Attribution 4.0 International License, which permits unrestricted use, distribution, and reproduction in any medium, provided the original work is properly cited.}

Published by Madridge Publishers

\begin{abstract}
In this editorial we propose an approach for improving brain lymphatic flow in autism through lymphatic drainage massage that is a strategy successfully utilized in a variety of neurological conditions where lymph coming from the brain finds obstacles at the level of deep cervical nodes. We estimate that manual lymphatic massage at the level of deep cervical nodes improves brain lymphatic drainage possibly resulting in decrease of neuroinflammation that is a hallmark of autism. In addition to stimulating lymphatic drainage, we theorize that such an approach may work also as vagus nerve stimulation with the additional benefit of targeting the larynx in a manner similar to that outlined for laryngeal manual therapy. This therapy is utilized for the treatment of dysphonia, and its effects on speech, a function that is typically altered in autism, may contribute to the overall improvement of symptoms. We propose that this type of inexpensive, safe and accessible approach can be beneficial to autistic subjects and constitute a new and promising treatment. We are convinced that the described approach will play a pivotal role in future options for autism treatment, possibly in combination with other therapeutic approaches such as behavioral therapies or nutritional supplementation.
\end{abstract}

Keywords: Autism; Brain Lymphatic System; Massage; Vagus Nerve; Larynx.

\section{Introduction}

Autism is a disorder of brain development where genetic, environmental, metabolic and other unknown causes contribute to the onset and progression of the disease [1]. As odd as it may appear at first glance, the complexity of autism with its interconnections between different etiological agents is similar to that of cancer and the two diseases share more similarities than differences (for rev. on this topic, see Ruggiero and Pacini [2]). If etiology of autism is yet to be fully understood, its pathogenesis appears to be clearer also thanks to our previous work with the late Dr. Bradstreet.

Our contribution to the understanding of autism pathogenesis saw a major breakthrough in 2014 when we evidenced lesions in the brains of autistic subjects that we defined as cortical dysplasia; these lesions were associated with accumulation of extra-axial fluid, and we demonstrated a significant correlated between these findings and the severity of symptoms [3]. Based on these clinical observations, in 2015, we communicated a study where we suggested that cortical dysplasia and extra-axial fluid accumulation in autism correlated with, and were possibly caused by, impaired flow of lymph from the brain because of obstacles at the level of the deep cervical nodes [4]. According to this interpretation, restricted flow of lymph is associated with infective or inflammatory conditions at the level of the mouth, ears, nose and throat; these processes lead to engorgement of the deep cervical nodes, a circumstance that can be clinically evidenced by enlarged palpable nodes and ultrasonography [5] and engorgement obstructs the flow of lymph through the nodes. In other words, defective drainage of 
lymph from the brain through the meningeal lymphatics that drain in the deep cervical nodes would then be culpable for the cortical dysplasia and extra-axial fluid accumulation that we had evidenced in the brains of autistic children. These events may be responsible for the pathogenesis of the autism spectrum disorder since restricted drainage of brain lymph is responsible for neuro-inflammation [6] that is an almost universal finding in autism [7].

This hypothesis of ours is supported by the clinical case reports communicated by Matarazzo in 2002 [8], where the researcher observed a significant association between otorhinolaryngologic infections and autism. In this paper, the Author reports two cases of children who developed normally in the first three years, but, before the age of three, developed typical symptoms of autism that were associated with, and possibly due to, reactivation of a chronic otorhinolaryngologic infection. In one case, pharmacological treatment targeting the immune system performed in the first months after appearance of symptoms led to complete recovery; in the other case, where the child was treated six years later, the treatment targeting the immune system led to a limited, but decisive, improvement [8]. In the year 2002, the brain lymphatic system had not yet been described and it was thought that the brain was disconnected from general lymphatic circulation. However, based on today's knowledge, we can reinterpret those results and theorize that the antiinflammatory/immunosuppressive treatment performed by Matarazzo with ACTH led to clearance of the engorgement of the deep cervical nodes associated with otorhinolaryngologic infection, thus restoring the flow of lymph from the brain. The clinical case reports by Matarazzo, reinterpreted according to our rationale, are consistent with another observation that predates the characterization of the brain lymphatic system, that is, the clinical implication of cerebrospinal fluid stasis in the pathogenesis of autism spectrum disorder [9].

The approach that is described below has to goal to improve brain lymphatic flow in autistic subjects utilizing manual lymphatic drainage massage; this is a therapeutic approach that has been successfully utilized in several conditions where lymphatic circulation is obstructed by obstacles at the level of nodes. For example, manual lymphatic drainage is prescribed for lymphedema that follows breast cancer therapies [10]. A recent review on effectiveness and safety of manual lymphatic drainage in breast cancer patients led to the conclusion that such a treatment is safe and provides further advantages over other therapies aimed at decreasing swelling [10]. Consistent with our assumption, there are reports suggesting that massage improves the symptoms of autism [11] even though, in the paper by Jerger et al. the massage was not performed with the specific goal of improving the flow of lymph through the deep cervical nodes.

\section{Description of the approach based on manual lymphatic drainage}

The approach that is here described is based on manual massage and can be associated with pharmacological therapies or nutritional strategies tailored to the needs of each individual such as those described in Antonucci et al. [12]. The massage is performed using a novel, petroleumfree, cosmetic cream endowed with specific rheological and sensory properties developed for this scope. This cream contains vegetable oil, water, phosphatidylcholine from sunflower, chondroitin sulfate and a probiotic blend of Bifidobacteria and kefir grains (Sophia Flow, Woodinville, WA, USA). We chose this cream with specific rheological and sensory properties because it has been demonstrated that massage yields better clinical results with the use of ointments. For example, it has been demonstrated that massage improves the quality of sleep in adults and infants, and in this situation, the results are better with the use of oil versus no oil [13]. Consistent with an active role for oils or creams in determining efficacy of massage, moderate pressure massage with sunflower oil determined greater augmentation of weight in underweight preterm neonates [14]. The approach that we propose here begins with collecting a small amount of the cream, about a chickpea size, using a clean, disposable tool such as a plastic spoon. The cream is then rubbed between the hands until it warms so that its fluidity increases. The subject is then helped in overextending the neck with the head tilted as far back as possible. The therapist, or a parent, operates the massage with both hands, gently stroking the surface of the neck on the sides of the trachea with open palms and extended fingers. The therapist, or the parent, initiates the massage from the top, with the tip of the fingers caressing the mandible and, with light pressure, like brushing, moves the fingers down to the base of the neck. The movement of the massage has to be cranio-caudal, from top to bottom, with the hands joining as they go down so to execute also a laryngeal manual massage. When such a procedure is practiced at home, parents are advised to perform the massage before bedtime, every day.

\section{Discussion}

In the present communication, we describe an approach based on manual lymphatic drainage that has the goal of reestablishing the flow of lymph from the brain in autism. It is worth mentioning that, in addition to autism, this approach may prove useful in all neurological conditions where cerebrospinal fluid stasis is involved such as, for example, Alzheimer's disease. We theorize that the approach illustrated here may work also as vagus nerve stimulation concurrently with its effect on lymphatic drainage. The deep cervical nodes are anatomically positioned in proximity of the vagus nerve, and manual stimulation may affect both the lymphatics and the vagus nerve. Thus, it is well known that autism is characterized by low vagal activity that leads, in addition to other symptoms, to gaze aversion and flat facial expressions. As a consequence, vagal stimulation utilizing a massage technique such as the one here described, may prove instrumental in ameliorating the symptoms of autism [15]. In the case of the approach illustrated here, vagal stimulation is obtained together with lymphatic drainage; such a dual effect has the advantage of addressing 
two major pathogenetic mechanisms that contribute to the symptoms of autism. It is worth noting that this type of massage also involves the larynx as it happens in the case of laryngeal manual therapy for the treatment of dysphonia [16]. It is interesting to note that, despite the great number of papers published on autism, there are only eight published studies in PubMed retrievable using the Keywords "autism and larynx", and none using "autism and laryngeal manual therapy" (search performed in December 2018). The absence of studies on the potential role of laryngeal massage in autism is surprising since abnormality of speech, if not bona fide dysphonia, is a common trait of autism. There is one study that supports the notion of a role for larynx physiology and pathology in autism. This is a single case report illustrating the situation of a 14-year-old autistic male, who presented with untreatable vocal tics. He was operated with a surgical procedure designated lateralization (type IIB) thyroplasty. As anticipated by the therapists, the frequency of tics was significantly reduced and the patient showed significant improvement in his ability to have a dialogue with his peers. However, in addition to this improvement that appears to be strictly associated with the function of the larynx, the boy also significantly improved in areas such as participation in school activities, and eating habits with consequent improvement of his nutritional status. These observations seem to indicate that targeting the larynx yielded results that were not strictly limited to the function of the organ [17].

Based on preliminary observation, we are confident that a cream with specific rheological properties will prove essential in obtaining improvement of symptoms following manual lymphatic drainage at the level of the deep cervical nodes. This assumption of ours is supported by the results of other researchers who have observed how specific oily ointments improve the efficacy of massage, in particular in infants, and yield positive results that become substantial in just a few days. An example of these results was reported by Taheri et al. [18] who observed how a short course (five days) of moderate pressure sunflower oil massage significantly improved weight gain in preterm infants at the same time significantly reducing their permanence in neonatal intensive care units.

We anticipate that the manual lymphatic drainage described in this paper will have a pivotal role in future treatments for autism, possibly in combination with other therapeutic treatments, such as behavioral therapies, immunotherapy and nutritional approaches.

\section{Acknowledgements}

The Authors wish to express their gratitude for the human and scientific legacy of Dr. Bradstreet whose insight led to the development of the protocol here described.

\section{Conflict of Interest}

Nicola Antonucci is the founder of the Biomedical Centre for Autism Research and Treatment, a private clinic. Marco Ruggiero is the inventor of a number of supplements and, together with Stefania Pacini, developed the cream used for the manual lymphatic drainage described in this study.

\section{Advisory}

No information in this paper is presented by the authors as medical advice. Caregivers, researchers and interested parties should research all information given. Beginning any significant biomedical or other interventions that may impact physiology, or making any changes to an established regimen should be discussed with the patient's physician in advance. Standard of care for each pathology must be followed as well as rules and regulations established by Health Authorities of each Country.

\section{References}

1. Bhat S, Acharya UR, Adeli H, Bairy GM, Adeli A. Autism: cause factors, early diagnosis and therapies. Rev Neurosci. 2014; 25: 841-850. doi: 10.1515/revneuro-2014-0056

2. Ruggiero $M$, Pacini S. From neurology to oncology: what have in common autism and cancer? the role of oncogenes, immune system and microbiota. J Neurol Stroke. 2018; 166-172. doi: 10.15406/ jnsk.2018.08.00303

3. Bradstreet JJ, Pacini S, Ruggiero M. A New Methodology of Viewing Extra-Axial Fluid and Cortical Abnormalities in Children with Autism via Transcranial Ultrasonography. Front Hum Neurosci. 2014; 15(7): 934. doi: 10.3389/fnhum.2013.00934

4. Bradstreet JJ, Ruggiero M, Pacini S. Commentary: Structural and functional features of central nervous system lymphatic vessels. Front Neurosci. 2015; 22(9): 485. doi: 10.3389/fnins.2015.00485

5. Louveau A, Smirnov I, Keyes TJ, et al. Structural and functional features of central nervous system lymphatic vessels. Nature. 2015; 523: 337-341. doi: 10.1038/nature14432

6. Louveau A, Herz J, Alme MN, et al. CNS lymphatic drainage and neuroinflammation are regulated by meningeal lymphatic vasculature. Nat Neurosci. 2018; 21: 1380-1391. doi: 10.1038/s41593-018-0227-9

7. Siniscalco D, Bradstreet JJ, Cirillo A, Antonucci N. The in vitro GcMAF effects on endocannabinoid system transcriptionomics, receptor formation, and cell activity of autism-derived macrophages. J Neuroinflammation. 2014; 11: 78. doi: 10.1186/1742-2094-11-78

8. Matarazzo EB. Treatment of late onset autism as a consequence of probable autommune processes related to chronic bacterial infection. World J Biol Psychiatry. 2002; 3(3): 162-166.

9. Whedon JM, Glassey D. Cerebrospinal fluid stasis and its clinical significance. Altern Ther Health Med. 2009; 15(3): 54-60.

10. Ezzo J, Manheimer $E, M c N e e l y ~ M L$, et al. Manual lymphatic drainage for lymphedema following breast cancer treatment. Cochrane Database Syst Rev. 2015; 21(5): CD003475. doi: 10.1002/14651858.CD003475.pub2

11. Jerger KK, Lundegard L, Piepmeier A, et al. Neural mechanisms of qigong sensory training massage for children with Autism spectrum disorder: A feasibility study. Glob Adv Health Med. 2018; 5(7): 2164956118769006. doi: 10.1177/2164956118769006

12. Antonucci $N$, Cirillo A, Siniscalco D. Beneficial effects of palmitoylethanolamide on expressive language, cognition, and behaviors in autism: A report of two cases. Case Rep Psychiatry. 2015; 2015: 325061. doi: 10.1155/2015/325061

13. Field T, Gonzalez G, Diego M, Mindell J. Mothers massaging their newborns with lotion versus no lotion enhances mothers' and newborns' sleep. Infant Behav. 2016; 45: 31-37. doi: 10.1016/j.infbeh.2016.08.004

14. Fallah R, Akhavan Karbasi S, Golestan M, Fromandi M. Sunflower oil versus no oil moderate pressure massage leads to greater increases in weight in preterm neonates who are low birth weight. Early Hum Dev. 2013; 89(9): 769-772. doi: 10.1016/j.earlhumdev.2013.06.002 
15. Field T, Diego M. Vagal activity, early growth and emotional development Infant Behav Dev. 2008; 31(3): 361-373. doi: 10.1016/j.infbeh.2007.12.008

16. Mathieson L. The evidence for laryngeal manual therapies in the treatment of muscle tension dysphonia. Curr Opin Otolaryngol Head Neck Surg. 2011; 19(3): 171-176. doi: 10.1097/MOO.0b013e3283448f6c
17. Ahmed MM, Heckman WW, Dailey SH. Type IIB thyroplasty for phonic tics in a pediatric patient with autism spectrum disorder: a case report. J Voice. 2013; 27: 242-244. doi: 10.1016/j.jvoice.2012.10.015

18. Taheri PA, Goudarzi Z, Shariat M, Nariman S, Matin EN. The effect of a short course of moderate pressure sunflower oil massage on the weight gain velocity and length of NICU stay in preterm infants. Infant Behav Dev. 2018; 50: 22-27. doi: 10.1016/j.infbeh.2017.11.002 